\title{
INTEGRABILITY OF GEODESIC FLOWS AND ISOSPECTRALITY OF RIEMANNIAN MANIFOLDS
}

\author{
DOROTHEE SCHUETH
}

\begin{abstract}
We construct a pair of compact, eight-dimensional, two-step Riemannian nilmanifolds $M$ and $M^{\prime}$ which are isospectral for the Laplace operator on functions and such that $M$ has completely integrable geodesic flow in the sense of Liouville, while $M^{\prime}$ has not. Moreover, for both manifolds we analyze the structure of the submanifolds of the unit tangent bundle given by to maximal continuous families of closed geodesics with generic velocity fields. The structure of these submanifolds turns out to reflect the above (non)integrability properties. On the other hand, their dimension is larger than that of the Lagrangian tori in $M$, indicating a degeneracy which might explain the fact that the wave invariants do not distinguish an integrable from a nonintegrable system here. Finally, we show that for $M$, the invariant eight-dimensional tori which are foliated by closed geodesics are dense in the unit tangent bundle, and that both $M$ and $M^{\prime}$ satisfy the so-called Clean Intersection Hypothesis.
\end{abstract}

\section{INTRODUCTION}

The spectrum of a compact Riemannian manifold is defined as the collection of eigenvalues of the Laplace operator acting on functions, counted with multiplicities. Two manifolds are called isospectral if their spectra are equal.

Inverse spectral geometry deals with the question of how much information the spectrum of a manifold provides about its geometry. Classical tools for extracting geometrical information from the spectrum are asymptotic expansions of the singularities of the heat trace or the wave trace. The so-called heat invariants determine the dimension, the volume, the total scalar curvature and a series of other integrals depending on the curvature tensor of the manifold. The singularities of the wave trace are contained in the set of lengths of closed geodesics on the manifold. Asymptotic expansions of these singularities near such a length yield, under suitable nondegeneracy assumptions, geometric information on the set of closed geodesics of this length; see the fundamental article by Duistermaat and Guillemin [3] or, for example, S. Zelditch's article [10] for more detailed results under stronger assumptions.

Closed geodesics thus being at the focus of the wave invariants, it is natural to ask to which extent integrability properties of the dynamical system given by the geodesic flow of a Riemannian manifold are determined by spectral data. In the present article, we will, more precisely, answer the question whether complete integrability of the geodesic flow in the sense of Liouville is a property determined by the Laplace spectrum on functions.

Key words and phrases. Laplace operator, isospectral manifolds, geodesic flows, complete integrability. 2000 Mathematics Subject Classification. 58J53, 53D25.

The author was partially supported by DFG Sonderforschungsbereich 647 . 
A Riemannian manifold $M$ is said to have completely integrable geodesic flow in the sense of Liouville if there exist $n=\operatorname{dim} M$ first integrals $f_{1}, \ldots f_{n} \in C^{\infty}\left(T^{*} M\right)$ of the geodesic flow which Poisson commute and are functionally independent. These notions are defined as follows: Let $\omega$ be the canonical symplectic form on $T^{*} M$. For any smooth function $f$ on $T^{*} M$, define the associated Hamiltonian vectorfield $X_{f}$ by $d f=\omega\left(., X_{f}\right)$. Then two functions $f, h \in C^{\infty}\left(T^{*} M\right)$ Poisson commute if $\omega\left(X_{f}, X_{h}\right)=0$, or equivalently, if $f$ is constant along the integral curves of $X_{h}$. A set $\left\{f_{1}, \ldots, f_{n}\right\}$ of smooth functions on $T^{*} M$ is called functionally independent if $d f_{1} \wedge \ldots \wedge d f_{n} \neq 0$ on an open dense subset of $T^{*} M$.

Our main result is:

Theorem 3.10. There exists a pair of compact closed isospectral Riemannian manifolds $M, M^{\prime}$ such that $M$ has completely integrable geodesic flow, while $M^{\prime}$ does not have completely integrable geodesic flow.

In recent years, L. Butler has studied the question of complete integrability of geodesic flows on compact Riemannian nilmanifolds. These are manifolds of the form $(\Gamma \backslash N, g)$, where $N$ is a simply connected nilpotent Lie group, $\Gamma$ is a cocompact discrete subgroup of $N$, and $g$ is a Riemannian metric induced by a left invariant metric on $N$. In particular, Butler has established sufficient criteria both for complete integrability and lack of complete integrability for the case of two-step Riemannian nilmanifolds [1], 2]. Applying his results, together with a certain construction by C. Gordon and E. Wilson [8] of isospectral nilmanifolds, we find a pair of compact eight-dimensional two-step Riemannian nilmanifolds $M, M^{\prime}$ with the properties claimed in the above theorem.

As an aside, we will also see that there exist continuous isospectral families of compact two-step Riemannian nilmanifolds each of which has completely integrable geodesic flow; see Corollary 3.6.

For the manifold $M$ we will, apart from just applying Butler's integrability condition, explicitly present a set of eight Poisson commuting and functionally independent first integrals for the geodesic flow (see Lemma 4.4), following the lines of Butler's proof of his criterion in [1]. Note that these first integrals are $C^{\infty}$ but not analytic; in fact, complete integrability by commuting analytic first integrals is impossible for compact nilmanifolds which are not tori, due to a theorem by I. Taimanov [9].

Moreover, for any closed unit speed geodesic whose velocity field satisfies a certain genericity condition, we will analyze the structure of the maximal continuous family of closed orbits of the geodesic flow in the unit tangent bundle of $M$, resp. $M^{\prime}$, containing the orbit associated with the given geodesic; see Corollary 5.6. While the dimension of $M$ and $M^{\prime}$ is eight, these subsets of the unit tangent bundles turn out to be ninedimensional submanifolds. "Generically" one would expect, at least in the case of $M$, eight dimensional submanifolds instead, namely, fibers of the (singular) $T^{8}$ foliation of the unit tangent bundle whose existence is guaranteed by complete integrability of the geodesic flow. The fact that the dimension of the submanifolds foliated by generic closed geodesics is higher than expected provides some explanation for why the wave invariants can, in this example, not distinguish between a completely integrable geodesic flow and a nonintegrable one, in the sense that some degeneracy is present here. 
On the other hand, the structure of these submanifolds reflects in a nice way the (non)integrability properties of the geodesic flow: While in the case of $M$, the submanifolds under consideration can be decomposed into a one-parameter family of invariant $T^{8}$ fibers which are level sets of the first integrals and two singular $T^{7}$ fibers, the picture is topologically quite different in the case of $M^{\prime}$ : Here, the corresponding submanifolds cannot be decomposed into invariant $T^{8}$ fibers, but instead (again up to two singular fibers) into a one-parameter family of invariant fibers diffeomorphic to $H^{3} \times T^{5}$, where $H^{3}$ is a compact three-dimensional Heisenberg manifold (a certain two-step nilmanifold not diffeomorphic to a torus).

We will also show that for both $M$ and $M^{\prime}$, the set of initial vectors of unit speed closed geodesics satisfying the considered genericity condition is dense in the unit tangent bundle. In particular, the invariant $T^{8}$ fibers in the unit tangent bundle of $M$ which are level sets of the first integrals and are fibered by closed orbits are dense in the unit tangent bundle; see Corollary 5.8.

Finally, using a result by Ruth Gornet [4], we show in Remark 5.9 that the so-called Clean Intersection Hypothesis (a hypothesis always needed in order to establish a wave trace formula [3]), is satisfied for both $M$ and $M^{\prime}$. So the reason for why the wave trace fails to distinguish between the different integrability properties of the geodesic flows of $M$ and $M^{\prime}$ does not lie in a failure of the Clean Intersection Hypothesis, but must be subtler.

In fact, the main reason seems to lie in the phenomenon already mentioned above, namely, that the closed geodesics fill out larger dimensional families than Lagrangian tori. In section 8 of his excellent survey paper [11], Zelditch explains why the the most natural setting for the "Can one hear integrability?" problem or any positive conjecture in this respect is that of "simple clean length spectrum", where, in the context of a manifold with completely integrable geodesic flow, "simple" means that for any given length, the family of closed geodesics of that length forms a Lagrangian submanifold which should, moreover, be connected. The natural approach for showing that any Riemannian manifold isospectral to a manifold with these properties and completely integrable geodesic flow should again have integrable geodesic flow - this approach has indeed yielded results in dimension two, see [11] - is the following: The wave invariants determine the dimension of the submanifolds foliated by closed geodesics, and they determine certain properties of the geodesic flow on these submanifolds. The hope is that if these submanifolds are Lagrangian tori in the reference manifold, then the wave invariants would force them to be tori also in the second manifold, which in turn might be used to derive integrability of its geodesic flow.

However, as we have seen, the Lagrangian condition is certainly violated in our examples because the dimension of the submanifolds in question is larger than half the dimension of the cotangent bundle. Let us mention here, without discussing it in the paper, that the connectedness condition is not satisfied either: For a given length, there will in general be more than one continuous family of closed geodesics of that length.

The paper is organized as follows:

In Sections 2 and 3, we set out the necessary framework on two-step Riemannian nilmanifolds and present the results of Gordon and Wilson [8] and Butler [1], [2], respectively, 
which we need for our construction. In Example 2.6 we define the pair of Riemanian manifolds $M$ and $M^{\prime}$ whose isospectrality is established in Section 2, while their integrability vs. nonintegrability properties are proven in Section 3. In Section 4 we explicitly establish eight Poisson commuting, functionally independent first integrals of the geodesic flow of $M$. In Section 5 we analyze the geometry of the submanifolds of the unit tangent bundles of $M$ and $M^{\prime}$ which are foliated by maximal continuous families of closed geodesics satisfying a certain genericity condition. We conclude by proving the results mentioned above on the density of closed geodesics and on the Clean Intersection Hypothesis.

The author would like to thank Steve Zelditch for raising her attention to the question of whether complete integrability of the geodesic flow is spectrally determined, and for several inspiring conversations.

\section{ISOSPECTRAL TWO-STEP NILMANIFOLDS}

Let $\mathfrak{v}$ and $\mathfrak{z}$ be euclidean vector spaces, each endowed with a fixed inner product.

Definition 2.1. Given the above data, one associates with any linear map $j: \mathfrak{z} \rightarrow \mathfrak{s o}(\mathfrak{v})$ the following:

(i) The two-step nilpotent metric Lie algebra $\mathfrak{n}(j)$ with underlying vector space $\mathfrak{v} \oplus \mathfrak{z}$, whose inner product is given by letting $\mathfrak{v}$ and $\mathfrak{z}$ be orthogonal and taking the given inner product on each factor, and whose Lie bracket $[,]^{j}$ is defined by letting $\mathfrak{z}$ be central, $[\mathfrak{v}, \mathfrak{v}]^{j} \subseteq \mathfrak{z}$ and $\langle j(Z) X, Y\rangle=\left\langle Z,[X, Y]^{j}\right\rangle$ for all $X, Y \in \mathfrak{v}$ and $Z \in \mathfrak{z}$.

(ii) The two-step simply connected nilpotent Lie group $N(j)$ whose Lie algebra is $\mathfrak{n}(j)$, and the left invariant Riemannian metric $g(j)$ on $N(j)$ which coincides with the chosen inner product on $\mathfrak{n}(j)=T_{e} N(j)$.

\section{Notation and Remarks 2.2.}

(i) Note that the Lie group exponential map $\exp ^{j}: \mathfrak{n}(j) \rightarrow N(j)$ is a diffeomorphism because $N(j)$ is simply connected and nilpotent.

(ii) Since $N(j)$ is two-step nilpotent, the Campbell-Baker-Hausdorff formula implies that $\exp ^{j}(X) \cdot \exp ^{j}(Y)=\exp ^{j}\left(X+Y+\frac{1}{2}[X, Y]^{j}\right)$ for all $X, Y \in \mathfrak{n}(j)$.

(iii) In particular, if a lattice $\mathcal{G}$ in $\mathfrak{v} \oplus \mathfrak{z}$ has the property that $[\mathcal{G}, \mathcal{G}]^{j} \subseteq 2 \mathcal{G}$, then $\Gamma:=\exp ^{j}(\mathcal{G})$ is a discrete subgroup of $N(j)$; hence $\Gamma \backslash N(j)$, endowed with the metric induced by $g(j)$, is a two-step Riemannian nilmanifold. We denote the induced metric by $g(j)$ again. If, moreover, $\mathcal{G}$ has full rank, then $\Gamma$ is cocompact, and $(\Gamma \backslash N(j), g(j))$ is a compact two-step Riemannian nilmanifold.

(iv) If $\mathcal{L}$ is a cocompact lattice in $\mathfrak{z}$, then we denote by $\mathcal{L}^{*}:=\{Z \in \mathfrak{z} \mid\langle Z, \mathcal{L}\rangle \subseteq \mathbb{Z}\}$ the dual lattice, viewed as a lattice in $\mathfrak{z}$.

\section{Definition 2.3.}

(i) Two linear maps $j, j^{\prime}: \mathfrak{z} \rightarrow \mathfrak{s o}(\mathfrak{v})$ are called isospectral if for each $Z \in \mathfrak{z}$, the maps $j(Z), j^{\prime}(Z) \in \mathfrak{s o}(\mathfrak{v})$ are similar, that is, have the same eigenvalues (with multiplicities) in $\mathbb{C}$.

(ii) Two lattices in a euclidean vector space are called isospectral if the lengths of their elements, counted with multiplicities, coincide. 
We will use the following special version of a result from [8].

Proposition 2.4 (see [8] 3.2, 3.7, 3.8). Let $j, j^{\prime}: \mathfrak{z} \rightarrow \mathfrak{s o}(\mathfrak{v})$ be isospectral. Let $\mathcal{M}$ and $\mathcal{L}$ be cocompact lattices in $\mathfrak{v}$ and $\mathfrak{z}$, respectively. Assume that $[\mathcal{M}, \mathcal{M}]^{j}$ and $[\mathcal{M}, \mathcal{M}]^{j^{\prime}}$ are contained in $2 \mathcal{L}$. For each $Z \in \mathcal{L}^{*}$ assume that the lattices $\operatorname{ker}(j(Z)) \cap \mathcal{M}$ and $\operatorname{ker}\left(j^{\prime}(Z)\right) \cap \mathcal{M}$ are isospectral. Write $\Gamma(j):=\exp ^{j}(\mathcal{M}+\mathcal{L})$, and similarly for $j^{\prime}$. Then the compact Riemannian manifolds $(\Gamma(j) \backslash N(j), g(j))$ and $\left(\Gamma\left(j^{\prime}\right) \backslash N\left(j^{\prime}\right), g\left(j^{\prime}\right)\right)$ are isospectral for the Laplace operator on functions.

Remark 2.5. (i) In the situation of Proposition 2.4, note that for $Z \in \mathcal{L}^{*}$, the subspace $\langle Z\rangle^{\perp}$ of $\mathfrak{z}$ is rational with respect to $\mathcal{L}$, that is, its intersection with $\mathcal{L}$ is a cocompact lattice in this subspace. Using the assumption $[\mathcal{M}, \mathcal{M}]^{j} \subseteq 2 \mathcal{L} \subset \mathcal{L}$ and the fact that $\mathcal{M}$ has full rank in $\mathfrak{v}$, one concludes that the subspace $\left\{X \in \mathfrak{v} \mid[X, \mathfrak{v}]^{j} \subseteq\langle Z\rangle^{\perp}\right\}=\operatorname{ker}(j(Z))$ of $\mathfrak{v}$ is rational with respect to $\mathcal{M}$. Thus $\operatorname{ker}(j(Z)) \cap \mathcal{M}$ is actually a cocompact lattice in $\operatorname{ker}(j(Z))$, and similarly for $j^{\prime}$.

(ii) We indicate how to derive Proposition 2.4 from the cited results of [8].

Let $Z \in \mathcal{L}^{*} \backslash\{0\}$. Consider the compact two-step Riemannian nilmanifold $M_{Z}$ associated as in 2.1 and 2.2 with the euclidean spaces $\mathfrak{v}$ and $\langle Z\rangle$, the linear map from $\langle Z\rangle$ to $\mathfrak{s o}(\mathfrak{v})$ mapping $Z$ to $j(Z)$, and the lattices $\mathcal{M} \subset \mathfrak{v}$ and $\operatorname{proj}_{\langle Z\rangle} \mathcal{L} \subset\langle Z\rangle$, where $\operatorname{proj}_{\langle Z\rangle}$ denotes orthogonal projection onto $\langle Z\rangle$. Analogously define $M_{Z}^{\prime}$ using $j(Z)$ instead of $j^{\prime}(Z)$. ( $M_{Z}$ is actually the Riemannian submersion quotient of $(\Gamma(j) \backslash N(j), g(j))$ by the canonical action of the torus $\langle Z\rangle^{\perp} /\left(\langle Z\rangle^{\perp} \cap \mathcal{L}\right)$ on this manifold, and similarly for $j^{\prime}$.)

We first note that $M_{Z}$ and $M_{Z}^{\prime}$ are then isospectral by Proposition 3.7/Remark 3.8 of [8]. In fact, their base tori (the Riemannian submersion quotients by the action of the circle $\left.\langle Z\rangle /\left(\langle Z\rangle \cap \operatorname{proj}_{\langle Z\rangle} \mathcal{L}\right)\right)$ are both isometric to the torus $\mathfrak{v} / \mathcal{M}$ and thus isospectral, the maps $j(Z)$ and $j^{\prime}(Z)$ are similar, the lattice in $\operatorname{ker}\left(j^{(\prime)}(Z)\right) \oplus\langle Z\rangle$ is a sum of lattices in the two factors as required in Remark 3.8 of [8], and the lattices $\operatorname{ker}(j(Z)) \cap \mathcal{M}$ and $\operatorname{ker}\left(j^{\prime}(Z)\right) \cap \mathcal{M}$ are isospectral by assumption.

Finally, the manifold $M_{0}$, defined as the Riemannian submersion quotient of the manifold $(\Gamma(j) \backslash N(j), g(j))$ by the action of the torus $\mathfrak{z} / \mathcal{L}$, is isometric to the analogously defined manifold $M_{0}^{\prime}$ since both are isometric to $\mathfrak{v} / \mathcal{M}$; in particular, $M_{0}$ and $M_{0}^{\prime}$ are isospectral. Isospectrality of the pairs $M_{Z}$ and $M_{Z}^{\prime}$ for each $Z \in \mathcal{L}^{*}$ now implies, by Theorem 3.2 of [8], the isospectrality statement of Proposition 2.4.

We now give an example of a pair of isospectral manifolds arising from Proposition 2.4 with the property that, as we will show in Section 3, the geodesic flow on the first manifold is completely integrable, while the geodesic flow on the second manifold is not.

Example 2.6. Let $\operatorname{dim} \mathfrak{v}=5, \operatorname{dim} \mathfrak{z}=3$, and let $\left\{X_{i}, X_{j}, Y_{i}, Y_{j}, Y_{k}\right\}$ and $\left\{Z_{i}, Z_{j}, Z_{k}\right\}$ be orthonormal bases of $\mathfrak{v}$ and $\mathfrak{z}$, respectively. Define two Lie brackets [,] and [, ] on $\mathfrak{v} \oplus \mathfrak{z}$ as follows: For $a, b \in\{i, j, k\}$ with $a \neq b$ denote by $a b \in\{ \pm i, \pm j, \pm k\}$ the quaternionian product of $a$ and $b$, and for $c \in\{i, j, k\}$ write $Z_{-c}:=-Z_{c}$. Now let $\left[X_{a}, Y_{b}\right]=-\left[Y_{b}, X_{a}\right]:=Z_{a b}$ and $\left[X_{a}, X_{b}\right]^{\prime}:=Z_{a b},\left[Y_{a}, Y_{b}\right]^{\prime}:=Z_{a b}$ for all $a \neq b$ in $\{i, j, k\}$, ignoring any expressions containing $X_{k}$, and let all other brackets between basis elements be zero. Then $[]=,[,]^{j}$ and $[,]^{\prime}=[,]^{j^{\prime}}$, where $j, j^{\prime}: \mathfrak{z} \rightarrow \mathfrak{s o}(\mathfrak{v})$ are the linear maps for 
which $j\left(c_{i} Z_{i}+c_{j} Z_{j}+c_{k} Z_{k}\right)$ and $j^{\prime}\left(c_{i} Z_{i}+c_{j} Z_{j}+c_{k} Z_{k}\right)$ with $c_{i}, c_{j}, c_{k} \in \mathbb{R}$ are expressed, with respect to the given basis of $\mathfrak{v}$, by the matrices

$$
\left(\begin{array}{ccccc}
0 & 0 & 0 & -c_{k} & c_{j} \\
0 & 0 & c_{k} & 0 & -c_{i} \\
0 & -c_{k} & 0 & 0 & 0 \\
c_{k} & 0 & 0 & 0 & 0 \\
-c_{j} & c_{i} & 0 & 0 & 0
\end{array}\right) \quad \text { and } \quad\left(\begin{array}{ccccc}
0 & -c_{k} & 0 & 0 & 0 \\
c_{k} & 0 & 0 & 0 & 0 \\
0 & 0 & 0 & -c_{k} & c_{j} \\
0 & 0 & c_{k} & 0 & -c_{i} \\
0 & 0 & -c_{j} & c_{i} & 0
\end{array}\right), \quad \text { respectively. }
$$

Let $\mathcal{M}$ be the lattice in $\mathfrak{v}$ generated by the basis given above, and $\mathcal{L}$ be the lattice in $\mathfrak{z}$ generated by $\left\{\frac{1}{2} Z_{i}, \frac{1}{2} Z_{j}, \frac{1}{2} Z_{k}\right\}$; in particular, $[\mathcal{M}, \mathcal{M}]^{j}=[\mathcal{M}, \mathcal{M}]^{j^{\prime}}=2 \mathcal{L}$. Then, with notation as in $2.1,2.2$, 2.4, the associated manifolds $(\Gamma(j) \backslash N(j), g(j))$ and $\left(\Gamma\left(j^{\prime}\right) \backslash N\left(j^{\prime}\right), g\left(j^{\prime}\right)\right)$ are isospectral by Proposition 2.4 .

In fact, $j$ and $j^{\prime}$ are isospectral since the two matrices given above have the same characteristic polynomials $\lambda\left(\lambda+c_{k}^{2}\right)\left(\lambda+c_{i}^{2}+c_{j}^{2}+c_{k}^{2}\right)$. It remains to show that for each $Z \in \mathcal{L}^{*}$, the two lattices $\operatorname{ker}(j(Z)) \cap \mathcal{M}$ and $\operatorname{ker}\left(j^{\prime}(Z)\right) \cap \mathcal{M}$ are isospectral; we will actually see that they are isometric. For $c=\left(c_{i}, c_{j}, c_{k}\right) \in \mathbb{R}^{3}$ write $X_{c}:=c_{i} X_{i}+c_{j} X_{j}$, $Y_{c}:=c_{i} Y_{i}+c_{j} Y_{j}+c_{k} Y_{k}, Z_{c}:=c_{i} Z_{i}+c_{j} Z_{j}+c_{k} Z_{k}$. Then we have for all $c \in \mathbb{R}^{3}$ and, in particular, for all $c \in(2 \mathbb{Z})^{3}$ (that is, for $\left.Z_{c} \in \mathcal{L}^{*}\right)$ :

$$
\begin{aligned}
& \operatorname{ker}\left(j\left(Z_{c}\right)\right)=\operatorname{ker}\left(j^{\prime}\left(Z_{c}\right)\right)=\operatorname{span}\left\{Y_{c}\right\} \quad \text { if } c_{k} \neq 0, \\
& \operatorname{ker}\left(j\left(Z_{c}\right)\right)=\operatorname{span}\left\{X_{c}, Y_{i}, Y_{j}\right\}, \quad \operatorname{ker}\left(j^{\prime}\left(Z_{c}\right)\right)=\operatorname{span}\left\{X_{i}, X_{j}, Y_{c}\right\} \quad \text { if } c_{k}=0 \text { and } c \neq 0, \\
& \operatorname{ker}\left(j\left(Z_{c}\right)\right)=\operatorname{ker}\left(j^{\prime}\left(Z_{c}\right)\right)=\mathfrak{v} \quad \text { if } c=0 .
\end{aligned}
$$

In the first case, the two subspaces are equal, and thus the two lattices in question coincide. The same holds for the third case. In the second case, note that $Y_{c}=c_{i} Y_{i}+c_{j} Y_{j}$ since $c_{k}=0$; this and the fact that $\mathcal{M} \cap \operatorname{span}\left\{X_{i}, X_{j}, Y_{i}, Y_{j}\right\}$ is invariant under exchanging the $X$-with the $Y$-space shows isometry of the lattices in the two subspaces also in this case.

Remark 2.7. The metric Lie algebras $\mathfrak{n}$ and $\mathfrak{n}^{\prime}$ are not isomorphic. This implies [8] that the two manifolds $(\Gamma \backslash N, g)$ and $\left(\Gamma^{\prime} \backslash N^{\prime}, g^{\prime}\right)$ from Example 2.6 are not locally isometric.

\section{Complete integrability OF GeOdesic FlOWs ON TWO-STEP Nilmanifolds}

We are going to use results by L. Butler [1], [2] in order to show that the first of the two isospectral manifolds from Example 2.6 has completely integrable geodesic flow, while the second has not.

Definition 3.1. $\quad$ (i) A two-step nilpotent Lie algebra $\mathfrak{n}$ is called a Heisenberg-Reiter Lie algebra, shortly: HR Lie algebra, if there exists a vector space decomposition $\mathfrak{n}=\mathfrak{x} \oplus \mathfrak{y} \oplus \mathfrak{z}$ such that $[\mathfrak{n}, \mathfrak{n}] \subseteq \mathfrak{z},[\mathfrak{z}, \mathfrak{n}]=0,[\mathfrak{x}, \mathfrak{x}]=0$, and $[\mathfrak{y}, \mathfrak{y}]=0$. Such a decomposition is then called a presentation of $\mathfrak{n}$.

(ii) A presentation $\mathfrak{x} \oplus \mathfrak{y} \oplus \mathfrak{z}$ of an HR Lie algebra $\mathfrak{n}$ is called injective if there exists $c \in \mathfrak{z}^{*}$ such that $\left.c\right|_{[X, \mathfrak{y}]} \neq 0$ for all $X \in \mathfrak{x} \backslash\{0\}$.

Theorem 3.2 ([1], Theorem 2.22). Let $\mathfrak{n}$ be an HR Lie algebra admitting an injective presentation, and let $N$ be the associated simply connected Lie group. Assume that there exists a discrete, cocompact subgroup $\Gamma$ of $N$. Then for any such $\Gamma$ and any left invariant metric $g$ on $N$, the geodesic flow of $(\Gamma \backslash N, g)$ is completely integrable. 
Corollary 3.3. The manifold $(\Gamma(j) \backslash N(j), g(j))$ from Example 2.6 has completely integrable geodesic flow.

Proof. We use the notation from Example 2.6.

Let $\mathfrak{x}:=\operatorname{span}\left\{X_{i}, X_{j}\right\}$ and $\mathfrak{y}:=\operatorname{span}\left\{Y_{i}, Y_{j}, Y_{k}\right\}$. Since $\mathfrak{x}$ and $\mathfrak{y}$ are abelian, $\mathfrak{n}(j)=$ $\mathfrak{x} \oplus \mathfrak{y} \oplus \mathfrak{z}$ is an HR Lie algebra. Note that $\left.j\left(Z_{k}\right)\right|_{\mathfrak{x}}$ is injective, and hence $\left\langle Z_{k},[X, \mathfrak{y}]\right\rangle=$ $\left\langle j\left(Z_{k}\right) X, \mathfrak{y}\right\rangle \neq 0$ for all $X \in \mathfrak{x} \backslash\{0\}$. It follows that $\mathfrak{x} \oplus \mathfrak{y} \oplus \mathfrak{z}$ is an injective representation of $\mathfrak{n}(j)$, and thus the corollary follows from Theorem 3.2.

Remark 3.4. $\quad$ (i) It seems that the proof of Theorem 3.2 in [1] presupposes that the rational and the HR structure of the Lie algebra $\mathfrak{n}$ are in some sense compatible; more precisely: The proof given there of complete integrability of the geodesic flow of $(\Gamma \backslash N, g)$ works without any additional arguments if there exists an injective presentation $\mathfrak{x} \oplus \mathfrak{y} \oplus \mathfrak{z}$ of $\mathfrak{n}$ with the property that there exists a complement $\tilde{\mathfrak{x}}$ of $\mathfrak{z}$ in $\mathfrak{x} \oplus \mathfrak{z}$ and a complement $\tilde{\mathfrak{y}}$ of $\mathfrak{z}$ in $\mathfrak{y} \oplus \mathfrak{z}$ such that the union $\tilde{\mathfrak{x}} \cup \tilde{\mathfrak{y}} \cup \mathfrak{z}$ contains a set of vectors which is mapped to a generating set for $\Gamma$ by the Lie group exponential map. (Of course, $\tilde{\mathfrak{x}} \oplus \tilde{\mathfrak{y}} \oplus \mathfrak{z}$ is then itself an injective presentation of $\mathfrak{n}$.)

(ii) By our construction of $\Gamma(j)$ in Example 2.6 it is clear that the injective representation $\mathfrak{x} \oplus \mathfrak{y} \oplus \mathfrak{z}$ of $\mathfrak{n}(j)$ from the proof of Corollary 3.3 does satisfy the additional assumption just mentioned. Moreover, we will reconfirm complete integrability of the geodesic flow on $(\Gamma(j) \backslash N(j), g(j))$ in Section 4 directly: Guided by the proof of Theorem 3.2 in [1], we will explicitly establish eight commuting, functionally independent first integrals.

Remark 3.5. It is easy to find examples of continuous families of isospectral manifolds each of which has completely integrable geodesic flow, using Butler's above result and not the above construction from [8], but another construction by Gordon and Wilson [7, involving so-called almost-inner automorphisms. For example, let $\operatorname{dim} \mathfrak{v}=4, \operatorname{dim} \mathfrak{z}=2$, and let $\left\{X_{1}, X_{2}, Y_{1}, Y_{2}\right\}$ and $\left\{Z_{1}, Z_{2}\right\}$ be orthonormal bases of $\mathfrak{v}$ and $\mathfrak{z}$, respectively. Define a Lie bracket on the orthogonal sum $\mathfrak{n}:=\mathfrak{v} \oplus \mathfrak{z}$ by letting $\left[X_{1}, Y_{1}\right]=\left[X_{2}, Y_{2}\right]=Z_{1}$, $\left[X_{1}, Y_{2}\right]=Z_{2}$, and letting pairs of basis vectors commute if they do not occur in these three equations. Let $N$ be the associated simply connected Lie group, associated with the left invariant metric $g$ defined by the given inner product on $\mathfrak{n}$. Now consider the continuous family of lattices $\Gamma_{t}:=\exp \left(\mathcal{G}_{t}\right)$ in the associated simply connected Lie group $N$, where $\mathcal{G}_{t}:=\operatorname{span}\left\{X_{1}, X_{2}, Y_{1}, Y_{2}+t Z_{2}, \frac{1}{2} Z_{1}, \frac{1}{2} Z_{2}\right\}$. By [7], the family $\left(\Gamma_{t} \backslash N, g\right)$ is nontrivial and isospectral (even strongly isospectral, that is, also on $p$-forms for all $p$ ). Note that these manifolds are locally isometric to each other because it is just the lattice that changes. Using the automorphism $\Phi_{t}$ of $N$ whose differential on $\mathfrak{n}$ is given by $\operatorname{Id}+\left\langle., Y_{2}\right\rangle \cdot t Z_{2}$, we can instead view this family as the deformation of the metric on a fixed underlying manifold because $\left(\Gamma \backslash N, \Phi_{t}^{*} g\right)$ is isometric to $\left(\Gamma_{t} \backslash N, g\right)$.

Since $\mathfrak{x}:=\operatorname{span}\left\{X_{1}, X_{2}\right\}$ and $\mathfrak{y}:=\operatorname{span}\left\{Y_{1}, Y_{2}\right\}$ are abelian, $\mathfrak{n}$ is an HR Lie algebra. Moreover, $j\left(Z_{1}\right)$ is invertible; in particular, $\mathfrak{x} \oplus \mathfrak{y} \oplus \mathfrak{z}$ is an injective presentation of $\mathfrak{n}$. Letting $\tilde{\mathfrak{x}}:=\mathfrak{x}$ and $\tilde{\mathfrak{y}}:=\operatorname{span}\left\{Y_{1}, Y_{2}+t Z_{2}\right\}$ in Remark 3.4(ii), we see that Butler's integrability theorem applies; hence, each of the manifolds in this isospectral family has completely integrable geodesic flow. It is also an easy exercise to find six commuting first integrals 
for each of these manifolds, along the lines of Butler's proof, similarly to how we will do this in the next section for the first manifold from Example 2.6. So we can state:

Corollary 3.6. There exist continuous isospectral families of compact Riemannian manifolds each of which has completely integrable geodesic flow.

However, it remains an open question whether there might even exist a continuous isospectral deformation of a manifold with completely integrable geodesic flow to a manifold whose geodesic flow is nonintegrable. Note that in the family just constructed, the geodesic flows of the manifolds involved, even though they share the property of complete integrability, are not symplectically conjugate (i.e., conjugate by a symplectomorphism) to each other because any pair of compact two-step Riemannian nilmanifolds with symplectically conjugate geodesic flows must be isometric [6].

We now consider a sufficient nonintegrability criterion by Butler [2]:

Definition 3.7. Let $\mathfrak{n}$ be a two-step nilpotent Lie algebra.

(i) For $\lambda \in \mathfrak{n}^{*}$ let $\mathfrak{n}_{\lambda}:=\left\{X \in \mathfrak{n} \mid \operatorname{ad}_{X}^{*} \lambda=0\right\}=\left\{X \in \mathfrak{n}|\lambda|_{[X, \mathfrak{n}]}=0\right\}$.

(ii) $\lambda \in \mathfrak{n}^{*}$ is called regular if $\mathfrak{n}_{\lambda}$ has minimal dimension.

(iii) $\mathfrak{n}$ is called nonintegrable if there exists a dense open subset $U$ of $\mathfrak{n}^{*} \times \mathfrak{n}^{*}$ such that for each $(\lambda, \mu) \in U$, both $\lambda$ and $\mu$ are regular and $\left[\mathfrak{n}_{\lambda}, \mathfrak{n}_{\mu}\right]$ has positive dimension.

Theorem 3.8 ([2], Theorem 1.3). Let $\mathfrak{n}$ be a nonintegrable two-step nilpotent Lie algebra, and let $N$ be the associated simply connected Lie group. Assume that there exists a discrete, cocompact subgroup $\Gamma$ of $N$. Then for any such $\Gamma$ and any left invariant metric $g$ on $N$, the geodesic flow of $(\Gamma \backslash N, g)$ is not completely integrable.

Corollary 3.9. The manifold $\left(\Gamma\left(j^{\prime}\right) \backslash N\left(j^{\prime}\right), g\left(j^{\prime}\right)\right)$ from Example 2.6 does not have completely integrable geodesic flow.

Proof. We use the notation from Example 2.6, Letting $\mathfrak{n}^{\prime}:=\mathfrak{n}\left(j^{\prime}\right)$, we write elements of $\mathfrak{n}^{\prime}=\mathfrak{v} \oplus \mathfrak{z}$ in the form $V+Z$ with $V \in \mathfrak{v}$ and $Z \in \mathfrak{z}$. Then for $(V+Z)^{*}:=\langle V+Z,.\rangle \in \mathfrak{n}^{\prime *}$ we have $\mathfrak{n}_{(V+Z)^{*}}^{\prime}=\operatorname{ker}\left(j^{\prime}(Z)\right) \oplus \mathfrak{z}$. Thus $(V+Z)^{*}$ is regular if and only if $Z=Z_{c}$ for some $c \in \mathbb{R}^{3}$ with $c_{k} \neq 0$; in that case, $\mathfrak{n}_{(V+Z)^{*}}^{\prime}=\operatorname{span}\left\{Y_{c}\right\} \oplus \mathfrak{z}$ (see the discussion in Example 2.6). Moreover, if $\tilde{V} \in \mathfrak{v}$ and $\tilde{Z}=Z_{\tilde{c}}$ with $\tilde{c}_{k} \neq 0$, then

$$
\left[\mathfrak{n}_{(V+Z)^{*}}^{\prime}, \mathfrak{n}_{(\tilde{V}+\tilde{Z})^{*}}^{\prime}\right]=\operatorname{span}\left\{\left[Y_{c}, Y_{\tilde{c}}\right]^{j^{\prime}}\right\}
$$

which has positive dimension (namely, dimension one) if and only if $c$ and $\tilde{c}$ are linearly independent, or equivalently: if $Z$ and $\tilde{Z}$ are linearly independent. The set of pairs of vectors $(V+Z, \tilde{V}+\tilde{Z}) \in \mathfrak{n}^{\prime} \times \mathfrak{n}^{\prime}$ such that $Z$ and $\tilde{Z}$ are linearly independent and both have nonvanishing $Z_{k}$-component is obviously open and dense in $\mathfrak{n}^{\prime} \times \mathfrak{n}^{\prime}$. Using the identification of $\mathfrak{n}^{\prime}$ with $\mathfrak{n}^{\prime *}$ induced by $\langle$,$\rangle , one concludes that there is an open and dense subset U$ of $\mathfrak{n}^{\prime *} \times \mathfrak{n}^{\prime *}$ with the property required in Definition 3.7(iii). Thus $\mathfrak{n}^{\prime}$ is nonintegrable, and the corollary follows from Theorem 3.8 .

We now conclude our main result: 
Theorem 3.10. There exists a pair of isospectral compact closed Riemannian manifolds $M, M^{\prime}$ such that $M$ has completely integrable geodesic flow, while $M^{\prime}$ does not have completely integrable geodesic flow.

Proof. This follows immediately from Example 2.6] and Corollaries 3.3 and 3.9, letting $M:=(\Gamma(j) \backslash N(j), g(j))$ and $M^{\prime}:=\left(\Gamma\left(j^{\prime}\right) \backslash N\left(j^{\prime}\right), g\left(j^{\prime}\right)\right)$.

\section{EXPLICIT FIRST INTEGRALS}

In this section we will explicitly establish eight Poisson commuting first integrals for the geodesic flow of the first manifold $(\Gamma(j) \backslash N(j), g(j))$ from Example 2.6, using the ideas of the proof of Theorem 3.2 from [1] (see also Remark 3.4). We will do our computations on the tangent bundle rather than on the cotangent bundle; these two are canonically identified by the euclidean metric induced by $g(j)$ on each tangent space.

Notation and Remarks 4.1. Let $\mathfrak{v}, \mathfrak{z}, j$ be data as in Definition 2.1, let $\mathfrak{n}:=\mathfrak{n}(j)$ be the associated two-step nilpotent Lie algebra with underlying vector space $\mathfrak{v} \oplus \mathfrak{z}$ and Lie bracket $[]:,=[,]^{j}$, let $N:=N(j)$ be the associated simply connected Lie group, $g:=g^{j}$ be the associated left invariant metric on $N$, and $\exp :=\exp ^{j}: \mathfrak{n} \rightarrow N$ be the Lie group exponential map.

(i) We denote elements of $\mathfrak{n}$ in the form $V+Z$ with $V \in \mathfrak{v}$ and $Z \in \mathfrak{z}$, and we denote elements of $N$ in the form $(v, z):=\exp (v+z)$ with $v \in \mathfrak{v}$ and $z \in \mathfrak{z}$. By the Campbell-Baker-Hausdorff formula we have

$$
(v, z) \cdot(\bar{v}, \bar{z})=\left(v+\bar{v}, z+\bar{z}+\frac{1}{2}[v, \bar{v}]\right) .
$$

(ii) For $a \in N$, we denote left multiplication by $a$ by $L_{a}: N \rightarrow N$. We identify the tangent bundle $T N$ with $N \times \mathfrak{n}$ using left translation; that is, we write $X \in T_{(v, z)} N$ in the form $\left((v, z), L_{(v, z) *}^{-1} X\right) \in N \times \mathfrak{n}$. Note that for $a \in N$ we have

$$
L_{a *}((v, z), X)=\left(L_{a}(v, z), X\right) .
$$

Lemma 4.2. [5] In the situation of [4.1, let $\gamma: \mathbb{R} \rightarrow N$ be a geodesic in $(N, g)$. Write $\dot{\gamma}(t)=((v(t), z(t)), V(t)+Z(t))$ with $v(t), V(t) \in \mathfrak{v}$ and $z(t), Z(t) \in \mathfrak{z}$. Then the following geodesic equations hold:

$$
\begin{gathered}
\dot{V}(t)=j(Z(t)) V(t), \quad \dot{Z}(t)=0, \\
\dot{v}(t)=V(t), \quad \dot{z}(t)=Z(t)+\frac{1}{2}[v(t), V(t)] ;
\end{gathered}
$$

hence $Z(t) \equiv Z(0)=: Z$ and

$$
\begin{gathered}
V(t)=e^{t j(Z)} V(0), \\
\dot{v}(t)=e^{t j(Z)} V(0), \quad \dot{z}(t)=Z+\frac{1}{2}[v(t), V(t)] .
\end{gathered}
$$

Notation and Remarks 4.3. In the following, let $\mathfrak{v}, \mathfrak{z}$ be as in Example 2.6, and let $j: \mathfrak{z} \rightarrow \mathfrak{s o}(\mathfrak{v})$ be the first of the two maps considered there. 
(i) For $Z \in \mathfrak{z}$ with $Z=Z_{c}=c_{i} Z_{i}+c_{j} Z_{j}+c_{k} Z_{k}$ let

$$
\begin{gathered}
E_{1}(Z):=c_{i} X_{i}+c_{j} X_{j}, \quad E_{2}(Z):=-c_{j} Y_{i}+c_{i} Y_{j} \\
E_{3}(Z):=|c|\left(c_{j} X_{i}-c_{i} X_{j}\right), \quad E_{4}(Z):=c_{k}\left(c_{i} Y_{i}+c_{j} Y_{j}\right)-\left(c_{i}^{2}+c_{j}^{2}\right) Y_{k}
\end{gathered}
$$

and

$$
Y(Z):=Y_{c}=c_{i} Y_{i}+c_{j} Y_{j}+c_{k} Y_{k} .
$$

Note that we then have

$$
\begin{gathered}
j(Z) E_{1}(Z)=c_{k} E_{2}(Z), \quad j(Z) E_{2}(Z)=-c_{k} E_{1}(Z), \\
j(Z) E_{3}(Z)=|c| E_{4}(Z), \quad j(Z) E_{4}(Z)=-|c| E_{3}(Z), \\
j(Z) Y(Z)=0 .
\end{gathered}
$$

Thus, for generic $Z$ (namely, with $|c|>\left|c_{k}\right|>0$ ), the sets $\left\{E_{1}(Z), E_{2}(Z)\right\}$ and $\left\{E_{3}(Z), E_{4}(Z)\right\}$ are bases of the eigenspaces associated with the eigenvalues $-c_{k}^{2}$ and $-|c|^{2}$ of $j(Z)^{2}$, respectively, and $Y(Z)$ spans the zero eigenspace of $j(Z)$.

(ii) Let $\mathfrak{x}:=\operatorname{span}\left\{X_{i}, X_{j}\right\}, \mathfrak{y}:=\operatorname{span}\left\{Y_{i}, Y_{j}, Y_{k}\right\}$ as in the proof of Corollary 3.3. For $Z \in \mathfrak{z}$ with $Z=c_{i} Z_{i}+c_{j} Z_{j}+c_{k} Z_{k}$ and $c_{k} \neq 0$ we denote by $C(Z): \mathfrak{y} \rightarrow \mathfrak{x}$ the linear map whose matrix with respect to the given bases of $\mathfrak{y}$ and $\mathfrak{x}$ is

$$
\left(c_{k}|c|^{2}\right)^{-1}\left(\begin{array}{ccc}
-c_{i} c_{j} & c_{i}^{2}+c_{k}^{2} & -c_{j} c_{k} \\
-c_{j}^{2}-c_{k}^{2} & c_{i} c_{j} & c_{i} c_{k}
\end{array}\right)
$$

Note that we then have

$$
\left.C(Z) \circ j(Z)\right|_{\mathfrak{x}}=\operatorname{Id}_{\mathfrak{x}}
$$

In fact, $C(Z)$ is just $\left.\left(\left.\left.j(Z)\right|_{\mathfrak{y}} j(Z)\right|_{\mathfrak{x}}\right)^{-1} j(Z)\right|_{\mathfrak{y}}$.

(iii) Let $\varphi \in C^{\infty}(\mathbb{R})$ be the map $x \mapsto e^{-1 / x^{2}}$ with $\varphi(0):=0$. Define $\Phi: \mathfrak{z} \rightarrow \mathbb{R}$ by $\Phi\left(c_{i} Z_{i}+c_{j} Z_{j}+c_{k} Z_{k}\right):=\varphi\left(c_{k}|c|^{2}\right)$.

(iv) For $V \in \mathfrak{v}=\mathfrak{x} \oplus \mathfrak{y}$ denote by $V_{\mathfrak{x}}$ and $V_{\mathfrak{y}}$ the components of $V$ in $\mathfrak{x}$ and $\mathfrak{y}$, respectively.

Lemma 4.4. We use Notation [4.1. Then in the situation of 4.3, each of the following eight functions on $T N$ is a smooth first integral of the geodesic flow on $(N, g):=$ $(N(j), g(j))$ and is invariant under the left action of $\Gamma:=\Gamma(j)$ from Example [2.6:

$$
\begin{aligned}
& q^{W}: T N \ni((v, z), V+Z) \mapsto\langle Z, W\rangle \in \mathbb{R} \quad \text { with } W \in\left\{Z_{i}, Z_{j}, Z_{k}\right\}, \\
& h_{1}: T N \ni((v, z), V+Z) \mapsto\left\langle V, E_{1}(Z)\right\rangle^{2}+\left\langle V, E_{2}(Z)\right\rangle^{2} \in \mathbb{R}, \\
& h_{2}: T N \ni((v, z), V+Z) \mapsto\left\langle V, E_{3}(Z)\right\rangle^{2}+\left\langle V, E_{4}(Z)\right\rangle^{2} \in \mathbb{R}, \\
& k: T N \ni((v, z), V+Z) \mapsto\langle V, Y(Z)\rangle \in \mathbb{R}, \\
& f^{X}: T N \ni((v, z), V+Z) \mapsto\left\{\begin{array}{l}
0 \in \mathbb{R} \text { if } Z=Z_{c} \text { with } c_{k}=0, \\
\Phi(Z) \sin \left(2 \pi\left\langle X, v_{\mathfrak{x}}-C(Z) V_{\mathfrak{y}}\right\rangle\right) \in \mathbb{R}, \text { else, } \\
\text { with } X \in\left\{X_{i}, X_{j}\right\} .
\end{array}\right.
\end{aligned}
$$

In particular, each of these functions descends to a first integral of the geodesic flow on $(\Gamma \backslash N, g)$. 
Proof. Smoothness of the above functions is immediate from their construction. Note that $q^{W}, h_{1}, h_{2}$, and $k$ are invariant under the left action of $N$ on $T N$. Moreover, if $a=(\bar{v}, \bar{z}) \in \Gamma$ then $\bar{v}$ is an integer combination of the basis vectors; hence

$$
f^{X}\left(L_{a *}((v, z), V+Z)\right)=f^{X}\left(\left(\bar{v}+v, \bar{z}+z+\frac{1}{2}[\bar{v}, v]\right), V+Z\right)=f^{X}((v, z), V+Z)
$$

for $X \in\left\{X_{i}, X_{j}\right\}$ because $\left\langle X, v_{\mathfrak{x}}\right\rangle$ differs from $\left\langle X, v_{\mathfrak{x}}+\bar{v}_{\mathfrak{x}}\right\rangle$ by an integer.

It remains to show that each of the eight functions is invariant under the geodesic flow. Let $\gamma$ be a geodesic in $(N, g)$ and write $\gamma(t)=((v(t), z(t)), V(t)+Z(t))$. Then $Z(t)=: Z$ is constant by Lemma 4.2, in particular, $q^{W} \circ \dot{\gamma}$ is constant. Always using 4.2 and the equations (11), we observe:

$$
\begin{aligned}
\left(h_{1} \circ \dot{\gamma}\right)^{\prime}(t) & =2\left\langle V(t), E_{1}(Z)\right\rangle\left\langle j(Z) V(t), E_{1}(Z)\right\rangle+2\left\langle V(t) E_{2}(Z)\right\rangle\left\langle j(Z) V(t), E_{2}(t)\right\rangle \\
& =-2\left\langle V(t), E_{1}(Z)\right\rangle\left\langle V(t), c_{k} E_{2}(Z)\right\rangle-2\left\langle V(t), E_{2}(t)\right\rangle\left\langle V(t),-c_{k} E_{1}(Z)\right\rangle=0,
\end{aligned}
$$

and similarly for $h_{2}$. Moreover,

$$
(k \circ \dot{\gamma})^{\prime}(t)=\langle j(Z) V(t), Y(Z)\rangle=-\langle V(t), j(Z) Y(Z)\rangle=0 .
$$

Finally, noting that $\frac{d}{d t} V(t)_{\mathfrak{y}}=(j(Z) V(t))_{\mathfrak{y}}=j(Z) V(t)_{\mathfrak{x}}$, we have

$$
\left(f^{X} \circ \dot{\gamma}\right)^{\prime}(t)=\Phi(Z) \cos \left(2 \pi\left\langle X, v(t)_{\mathfrak{x}}-C(Z) V(t)_{\mathfrak{y}}\right) \cdot 2 \pi\left\langle X, V(t)_{\mathfrak{x}}-C(Z) j(Z) V(t)_{\mathfrak{x}}\right\rangle=0\right.
$$

if $Z=Z_{c}$ with $c_{k} \neq 0$; if $c_{k}=0$ then $\left(f^{X} \circ \dot{\gamma}\right)(t) \equiv 0$ by definition.

Lemma 4.5. (i) The eight first integrals from Lemma 4.4(i) are functionally independent, and

(ii) they Poisson commute with each other.

Proof. (i) Note that with respect to the left invariant Riemannian product metric $g \times\langle$, on $T N \cong N \times \mathfrak{n}$, the gradients of the functions $q^{Z_{i}}, q^{Z_{j}}, q^{Z_{k}}, h_{1}, h_{2}, k$ are all tangent to the second factor. The gradients of $q^{Z_{i}}, q^{Z_{j}}, q^{Z_{k}}$ at $((v, z), V+Z) \in T N$ are just

$$
\left(0, Z_{i}\right),\left(0, Z_{j}\right),\left(0, Z_{k}\right) \in T_{(v, z)} N \oplus T_{V+Z} \mathfrak{n} \cong T_{(v, z)} N \oplus \mathfrak{n} .
$$

The gradients of $h_{1}, h_{2}, k$ at $((v, z), V+Z)$, viewed as elements of $T_{(v, z)} N \oplus \mathfrak{n}$, are of the form

$$
\begin{aligned}
& \left(0,2\left\langle V, E_{1}(Z)\right\rangle E_{1}(Z)+2\left\langle V, E_{2}(Z)\right\rangle E_{2}(Z)+W\right) \\
& \left(0,2\left\langle V, E_{3}(Z)\right\rangle E_{3}(Z)+2\left\langle V, E_{4}(Z)\right\rangle E_{4}(Z)+\tilde{W}\right)
\end{aligned}
$$

$$
(0, Y(Z)+U)
$$

respectively, where $W, \tilde{W}, U$ are in $\mathfrak{z}$. If $Z=Z_{c}$ with $|c|>\left|c_{k}\right|>0$, and if $V$ is not orthogonal to any of the subspaces $\operatorname{span}\left\{E_{1}(Z), E_{2}(Z)\right\}, \operatorname{span}\left\{E_{3}(Z), E_{4}(Z)\right\}$, and $\operatorname{span}\{Y(Z)\}$, then these six gradients are obviously linearly independent (recall 4.3(i)). Moreover, for these $Z$, the gradient of $f^{X_{i}}$ at the point $((v, z), V+Z)$ is of the form

$$
(0, W)+\Phi(Z) \cos \left(2 \pi\left\langle X_{i}, v_{\mathfrak{x}}-C(Z) V_{\mathfrak{y}}\right\rangle\right) \cdot 2 \pi\left(L_{(v, z) *} X_{i},-{ }^{t} C(Z) X_{i}\right) \in T_{(v, z)} N \oplus \mathfrak{n}
$$

with some $W \in \mathfrak{z}$, and similarly for $X_{j}$. Since $L_{(v, z) *} X_{i}$ and $L_{(v, z) *} X_{j}$ are linearly independent, it follows that the set of points in $T N$ at which all eight gradients are linearly independent is open and dense in $T N$. 
(ii) The symplectic form $\omega$ on $T N$, after identification with $T^{*} N$ by the left invariant metric $g$, is given at the point $((v, z), V+Z) \in T N \cong N \times \mathfrak{n}$ by

$$
\begin{aligned}
\omega_{((v, z), V+Z)}\left(\left(L_{(v, z) *} A, B\right),\left(L_{(v, z) *} \tilde{A}, \tilde{B}\right)\right) & =\langle B, \tilde{A}\rangle-\langle A, \tilde{B}\rangle-\langle V+Z,[A, \tilde{A}]\rangle \\
& =\langle B, \tilde{A}\rangle-\langle A, \tilde{B}\rangle-\left\langle j(Z) A_{\mathfrak{v}}, \tilde{A}_{\mathfrak{v}}\right\rangle
\end{aligned}
$$

for all $A, B, \tilde{A}, \tilde{B} \in \mathfrak{n}$, where $A_{\mathfrak{v}}$ denotes the $\mathfrak{v}$-component of $A$ in $\mathfrak{n}=\mathfrak{v} \oplus \mathfrak{z}$. For any $f \in C^{\infty}(T N)$, the Hamiltonian vectorfield $X_{f}$ is characterized by $\langle., \operatorname{grad} f\rangle=d f=$ $\omega\left(., X_{f}\right)$. This and the above formula for $\omega$ shows that if the gradient of $f$ at the point $((v, z), V+Z) \in T N$ is $\left(L_{(v, z) *} B, A\right)$, then $X_{f}$ at this point is

$$
\left(L_{(v, z) *} A,-B+j(Z) A_{\mathfrak{v}}\right) \text {. }
$$

Therefore, the Hamiltonian vectorfields of the functions $q^{Z_{i}}, h_{1}, h_{2}, k, f^{X_{i}}$ at the point $((v, z), V+Z)$ are of the form

$$
\begin{gathered}
\left(Z_{i}, 0\right), \\
\left(*, 2\left\langle V, E_{1}(Z)\right\rangle j(Z) E_{1}(Z)+2\left\langle V, E_{2}(Z)\right\rangle j(Z) E_{2}(Z)\right), \\
\left(*, 2\left\langle V, E_{3}(Z)\right\rangle j(Z) E_{3}(Z)+2\left\langle V, E_{4}(Z)\right\rangle j(Z) E_{4}(Z)\right), \\
(*, j(Z) Y(Z))=(*, 0), \\
(W, 0)+\Phi(Z) \cos \left(2 \pi\left\langle X_{i}, v_{\mathfrak{x}}-C(Z) V_{\mathfrak{y}}\right\rangle\right) \cdot 2 \pi\left(-L_{(v, z) *}{ }^{t} C(Z) X_{i},-X_{i}-j(Z)^{t} C(Z) X_{i}\right) \\
=\left(W-\Phi(Z) \cos (\ldots) \cdot 2 \pi L_{(v, z) *}{ }^{t} C(Z) X_{i}, 0\right),
\end{gathered}
$$

with some $W \in \mathfrak{z}$, where the last equality follows from

$$
-j(Z)^{t} C(Z) X_{i}={ }^{t}\left(\left.j(Z)\right|_{\mathfrak{x}}\right)^{t} C(Z) X_{i}=X_{i}
$$

The formulas for $q^{Z_{j}}, q^{Z_{k}}, f^{X_{j}}$ are analogous. Thus, the second components of the Hamiltonian vectorfields of the functions $q^{W}, k, f^{X}$ vanish. Since the functions $q^{W}, h_{1}, h_{2}, k$ depend only on the second component of $((v, z), V+Z)$, it follows immediately that the latter functions Poisson commute with the $q^{W}, k, f^{X}$. It only remains to show that $\left\{h_{1}, h_{2}\right\}=0$ and $\left\{f^{X_{i}}, f^{X_{j}}\right\}=0$ : The derivative of $h_{2}$ in direction of $X_{h_{1}}$ vanishes because the second component of $X_{h_{1}}$ at $((v, z), V+Z)$ is in $\operatorname{span}\left\{E_{1}(Z), E_{2}(Z)\right\}$ and thus orthogonal to $E_{3}(Z)$ and $E_{4}(Z)$; the derivative of $f^{X_{j}}$ in direction of $X_{f^{X_{i}}}$ vanishes because ${ }^{t} C(Z) X_{i} \in \mathfrak{y}$ has vanishing $\mathfrak{x}$-component.

\section{Structure of Submanifolds Foliated By GeneriC ClOSED ORBits}

In this section, we will describe the submanifolds of the unit tangent bundles foliated by continuous families of closed geodesics in the two manifolds from Example 2.6. We will consider only families most of whose geodesics have velocity vectors satisfying a certain genericity condition. The result (Corollary [5.6) will nicely reflect the (non)integrability properties of the geodesic flows established in Section 3.

Notation 5.1. Let $(\Gamma \backslash N, g):=(\Gamma(j) \backslash N(j), g(j))$ and $\left(\Gamma^{\prime} \backslash N^{\prime}, g^{\prime}\right):=\left(\Gamma\left(j^{\prime}\right) \backslash N\left(j^{\prime}\right), g\left(j^{\prime}\right)\right)$ be the two manifolds from Example 2.6. Let $\gamma: \mathbb{R} \rightarrow\left(N^{(\prime)}, g^{(\prime)}\right)$ be a geodesic. Recalling Notation 4.1, write $\dot{\gamma}(0)=(\gamma(0), V+Z)$ for some $V \in \mathfrak{v}, Z=Z_{c} \in \mathfrak{z}$. If $|c|>\left|c_{k}\right|>0$ then we write $V=V_{c_{k}}+V_{|c|}+V_{0}$ where $V_{\lambda}$ denotes the component of $V$ in the $\left(-\lambda^{2}\right)$-eigenspace 
of $j^{(\prime)}(Z)^{2}$. In what follows, we will restrict our attention to geodesics $\gamma$ with "generic" velocity fields; by this, we mean that the vectors $Z=Z_{c}$ and $V$ satisfy the following genericity condition:

$$
|c|>\left|c_{k}\right|>0 \text { and } V_{c_{k}} \neq 0, V_{|c|} \neq 0, V_{0} \neq 0 \text {. }
$$

Note that by the geodesic equations 4.2, this property is invariant under the geodesic flow; so all $\dot{\gamma}(t)$ will satisfy the corresponding condition if $\dot{\gamma}(0)$ does so. Moreover, note that the set of tangent vectors satisfying this genericity condition is open and dense in the tangent bundle $T N^{(\prime)}$.

Remark 5.2. Let $\gamma: \mathbb{R} \rightarrow\left(N^{(\prime)}, g^{(\prime)}\right)$ be a geodesic, and let $\tau>0$. Then $\gamma$ will descend to a $\tau$-periodic geodesic in the quotient manifold $\left(\Gamma^{(\prime)} \backslash N^{(\prime)}, g^{(\prime)}\right)$ if and only if

$$
a:=\gamma(\tau) \gamma(0)^{-1} \in \Gamma^{(\prime)} \text { and } \dot{\gamma}(\tau)=L_{a *} \dot{\gamma}(0)
$$

Thus, if $\dot{\gamma}(0)=(\gamma(0), V+Z)$, then a necessary condition for $\gamma$ to descend to a $\tau$-periodic geodesic is $\dot{\gamma}(\tau)=(\gamma(\tau), V+Z)$ with the same vector $V+Z \in \mathfrak{n}$. Assuming this condition and the genericity condition (2) for $V+Z$, we will in the following lemma compute the translational element $a=\gamma(\tau) \gamma(0)^{-1}$ in terms of $\tau, V+Z$, and $\gamma(0)$. We first supply some notation concerning eigenvectors of $j^{\prime}(Z)^{2}$, analogous to Notation 4.3 .

Notation and Remarks 5.3. Let $j^{\prime}: \mathfrak{z} \rightarrow \mathfrak{s o}(\mathfrak{v})$ be the second of the two maps from Example 2.6. For $Z \in \mathfrak{z}$ with $Z=Z_{c}=c_{i} Z_{i}+c_{j} Z_{j}+c_{k} Z_{k}$ let

$$
\begin{gathered}
E_{1}^{\prime}(Z):=X_{i}, \quad E_{2}^{\prime}(Z):=X_{j} \\
E_{3}^{\prime}(Z):=|c|\left(c_{j} Y_{i}-c_{i} Y_{j}\right), \quad E_{4}^{\prime}(Z):=c_{k}\left(c_{i} Y_{i}+c_{j} Y_{j}\right)-\left(c_{i}^{2}+c_{j}^{2}\right) Y_{k}
\end{gathered}
$$

and

$$
Y(Z):=Y_{c}=c_{i} Y_{i}+c_{j} Y_{j}+c_{k} Y_{k}
$$

Note that we then have

$$
\begin{gathered}
j^{\prime}(Z) E_{1}^{\prime}(Z)=c_{k} E_{2}^{\prime}(Z), \quad j^{\prime}(Z) E_{2}^{\prime}(Z)=-c_{k} E_{1}^{\prime}(Z), \\
j^{\prime}(Z) E_{3}^{\prime}(Z)=|c| E_{4}^{\prime}(Z), \quad j^{\prime}(Z) E_{4}^{\prime}(Z)=-|c| E_{3}^{\prime}(Z), \\
j^{\prime}(Z) Y(Z)=0 .
\end{gathered}
$$

Lemma 5.4. Let $V+Z \in \mathfrak{v} \oplus \mathfrak{z}$ satisfy the genericity condition (2). Let $\gamma: \mathbb{R} \rightarrow\left(N^{(\prime)}, g^{(\prime)}\right)$ be a geodesic with $\dot{\gamma}(0)=((v, z), V+Z)$, where $(v, z)=\gamma(0)$. Let $\tau>0$, and assume that $\dot{\gamma}(\tau)=(\gamma(\tau), V+Z)$. Then the translational element $a:=\gamma(\tau) \gamma(0)^{-1}$ is equal to

$$
\begin{aligned}
& \left(\tau V_{0}, \tau\left(1+\frac{\left|V_{\perp}\right|^{2}}{2|c|^{2}}\right) \cdot Z_{c}+\tau \beta\left(\alpha_{2}-\frac{c_{k}}{c_{i}^{2}+c_{j}^{2}}\left(x_{i} c_{i}+x_{j} c_{j}\right)\right) \cdot\left(-c_{j} Z_{i}+c_{i} Z_{j}\right)\right. \\
& \left.\quad+\tau\left(-\frac{\left|V_{c_{k}}\right|^{2}}{2 c_{k}|c|^{2}}+\beta\left(\alpha_{4}-\frac{1}{c_{i}^{2}+c_{j}^{2}}\left(x_{i} c_{j}-x_{j} c_{i}\right)\right)\right) \cdot\left(c_{k}\left(c_{i} Z_{i}+c_{j} Z_{j}\right)-\left(c_{i}^{2}+c_{j}^{2}\right) Z_{k}\right)\right)
\end{aligned}
$$


in $(N, g)$, respectively to

$$
\begin{aligned}
& \left(\tau V_{0}, \tau\left(1+\frac{\left|V_{\perp}\right|^{2}}{2|c|^{2}}\right) \cdot Z_{c}+\tau \beta\left(-|c| \alpha_{3}^{\prime}+y_{k}-\frac{c_{k}}{c_{i}^{2}+c_{j}^{2}}\left(y_{i} c_{i}+y_{j} c_{j}\right)\right) \cdot\left(-c_{j} Z_{i}+c_{i} Z_{j}\right)\right. \\
& \left.\quad+\tau\left(-\frac{\left|V_{c_{k}}\right|^{2}}{2 c_{k}|c|^{2}}+\beta\left(\alpha_{4}^{\prime}-\frac{1}{c_{i}^{2}+c_{j}^{2}}\left(y_{i} c_{j}-y_{j} c_{i}\right)\right)\right) \cdot\left(c_{k}\left(c_{i} Z_{i}+c_{j} Z_{j}\right)-\left(c_{i}^{2}+c_{j}^{2}\right) Z_{k}\right)\right)
\end{aligned}
$$

in $\left(N^{\prime}, g^{\prime}\right)$, where, using Notation 5.1(ii), 4.3, and 5.3, we write $Z=Z_{c}, V_{0}=\beta Y(Z)$, $V_{\perp}:=V_{c_{k}}+V_{|c|}=: \sum_{m=1}^{4} \alpha_{m}^{(\prime)} E_{m}^{(\prime)}(Z)$, and $v=x_{i} X_{i}+x_{j} X_{j}+y_{i} Y_{i}+y_{j} Y_{j}+y_{k} Y_{k}$. Moreover, we have $\tau c_{k} \in 2 \pi \mathbb{Z}$ and $\tau|c| \in 2 \pi \mathbb{Z}$.

Proof. By the geodesic equations 4.2 and our assumption on $\dot{\gamma}(\tau)$, we have $e^{\tau j(Z)} V=V$. By the genericity condition on $V$, this implies here that $e^{\tau j(Z)}=\operatorname{Id}_{\mathfrak{v}}$; in particular, $\tau c_{k}$ and $\tau|c|$ are in $2 \pi \mathbb{Z}$.

In addition, assume for the moment that $\gamma(0)$ equals $e=(0,0)$, the neutral element of $N^{(\prime)}$. In this situation, one sees either by using formulas from [5] or by direct integration using the geodesic equations 4.2 and our explicit knowledge of the action of $j^{(\prime)}(Z)$ on the three different eigenspaces of $j^{(\prime)}(Z)^{2}$ :

$$
\gamma(\tau)=\left(\tau V_{0}, \tau Z+\tau\left[V_{0}, j(Z)^{-1} V_{\perp}\right]+\frac{1}{2} \tau\left[j(Z)^{-1} V_{c_{k}}, V_{c_{k}}\right]+\frac{1}{2} \tau\left[j(Z)^{-1} V_{|c|}, V_{|c|}\right]\right)
$$

in $(N, g)$, and the analogous formula for $\left(N^{\prime}, g^{\prime}\right)$ with $j^{\prime}(Z)$ instead of $j(Z)$ and $[,]^{\prime}$ instead of $[$,$] , where j^{(\prime)}(Z)^{-1}$ denotes the inverse of $\left.j^{(\prime)}(Z)\right|_{\operatorname{span}\left\{V_{0}\right\}^{\perp}}$. Now if $\gamma(0)=$ $(v, z)$ is arbitrary, then $\bar{\gamma}:=L_{(v, z)}^{-1} \circ \gamma$ is a geodesic as just considered, with $\dot{\bar{\gamma}}(0)=$ $((0,0), V+Z)$. Then $\gamma(\tau) \gamma(0)^{-1}=(v, z) \bar{\gamma}(\tau)(v, z)^{-1}$. For any element $(\bar{v}, \bar{z}) \in N^{(\prime)}$, we have $(v, z)(\bar{v}, \bar{z})(v, z)^{-1}=\left(\bar{v}, \bar{z}+[v, \bar{v}]^{(\prime)}\right)$ by $4.1(\mathrm{i})$. Thus, by adding the term $\left[v, \tau V_{0}\right]$ to the $z$-component in the above formula, we get

$$
a=\left(\tau V_{0}, \tau Z+\tau\left[v, V_{0}\right]+\tau\left[V_{0}, j(Z)^{-1} V_{\perp}\right]+\frac{1}{2} \tau\left[j(Z)^{-1} V_{c_{k}}, V_{c_{k}}\right]+\frac{1}{2} \tau\left[j(Z)^{-1} V_{|c|}, V_{|c|}\right]\right)
$$

in $(N, g)$, and the analogous formula for $\left(N^{\prime}, g^{\prime}\right)$.

The rest of the proof consists in evaluating this formula in $(N, g)$ and $\left(N^{\prime}, g^{\prime}\right)$, respectively, using the definition of the Lie brackets $[$,$] and [,]^{\prime}$ and the facts $V_{0}=\beta Y(Z)=$ $\beta Y_{c}, V_{\perp}=V_{c_{k}}+V_{|c|}, V_{c_{k}}=\alpha_{1}^{(\prime)} E_{1}^{(\prime)}(Z)+\alpha_{2}^{(\prime)} E_{2}^{(\prime)}(Z), V_{|c|}=\alpha_{3}^{(\prime)} E_{3}^{(\prime)}(Z)+\alpha_{4}^{(\prime)} E_{4}^{(\prime)}(Z)$, $j^{(\prime)}(Z)^{-1} V_{c_{k}}=-\frac{\alpha_{1}^{(\prime)}}{c_{k}} E_{2}^{(\prime)}(Z)+\frac{\alpha_{2}^{(\prime)}}{c_{k}} E_{1}^{(\prime)}(Z)$ and similarly for $j^{(\prime)}(Z)^{-1} V_{|c|}$; for developing the resulting $z$-component into the claimed form it is moreover useful to note that $Z_{k}=\frac{c_{k}}{|c|^{2}} Z_{c}-\frac{1}{|c|^{2}}\left(c_{k}\left(c_{i} Z_{i}+c_{j} Z_{j}\right)-\left(c_{i}^{2}+c_{j}^{2}\right) Z_{k}\right)$. The computation is a little tedious, but straightforward; we spare the reader the details here.

Remark 5.5. Note that by Lemma 5.4, all translational elements belonging to closed geodesics with velocity fields satisfying the genericity condition (2) are elements of the codimension two submanifold $\exp (\mathfrak{y} \oplus \mathfrak{z}) \subset \exp \left(\mathfrak{n}^{(\prime)}\right)=N^{(\prime)}$. So, only a quite special type of free homotopy classes in $\Gamma^{(\prime)} \backslash N^{(\prime)}$ contains closed geodesics with generic velocity fields in the above sense. This, however, should not lead to doubts as to whether the notion of genericity is out of place here. The set of free homotopy classes is discrete 
anyway, and there is no notion of genericity within this set. Our genericity condition concerns only the velocity vectors of the closed geodesics, as elements of the manifold $T N^{(\prime)}$. It is a common phenomenon in compact Riemannian nilmanifolds that "generic" closed geodesics belong only to a quite special set of free homotopy classes. For example, in a compact Riemannian Heisenberg manifold (or, more generally, in any nonsingular two-step Riemannian nilmanifold), all "generic" closed geodesics belong to central free homotopy classes, while the other free homotopy classes contain only geodesics with very special velocity fields.

Corollary 5.6. Under the assumptions of Lemma 5.4, assume that $a \in \Gamma^{(\prime)}$; in particular, $\left.\gamma\right|_{[0, \tau]}$ descends to a closed geodesic $\hat{\gamma}$ in $\left(\Gamma^{(\prime)} \backslash N^{(\prime)}, g^{(\prime)}\right)$. Moreover, assume that $\gamma$ is a unit speed geodesic (i.e., $|V+Z|=1$ ). Then the velocity fields of the largest continuous family of closed unit speed geodesics containing $\hat{\gamma}$ foliate a submanifold $C$, resp. $C^{\prime}$, of the unit tangent bundle $S(\Gamma \backslash N)$, resp. $S\left(\Gamma^{\prime} \backslash N^{\prime}\right)$, with the following properties:

(i) $C$ is diffeomorphic to $T^{6} \times S^{3}$; it consists of a one-parameter family of submanifolds invariant under the geodesic flow and diffeomorphic to $T^{6} \times S^{1} \times S^{1}=T^{8}$, and two singular seven-dimensional fibers diffeomorphic to $T^{6} \times S^{1}=T^{7}$. The invariant $T^{8}$ fibers are level sets of the first integrals from Lemma 4.4. The above decomposition of $C$ arises from the decomposition of the $S^{3}$ factor into a one-parameter family of $T^{2}=S_{r}^{1} \times S_{\sqrt{1-r^{2}}}^{1}$ fibers and two singular $S^{1}$ fibers.

(ii) $C^{\prime}$ is diffeomorphic to $H^{3} \times T^{3} \times S^{3}$, where $H^{3}$ is a compact three-dimensional Heisenberg manifold (in particular, not diffeomorphic to $T^{3}$ ). $C^{\prime}$ consists of a one-parameter family of submanifolds invariant under the geodesic flow and diffeomorphic to $H^{3} \times T^{3} \times T^{2}=H^{3} \times T^{5}$, and two singular seven-dimensional fibers diffeomorphic to $H^{3} \times T^{4}$. This decomposition of $C^{\prime}$ arises from the corresponding decomposition of the $S^{3}$ factor.

Proof. Let $\hat{\gamma}_{s}$ be a continuous family of closed unit speed geodesics with $\hat{\gamma}_{0}=\hat{\gamma}$. For the time being, we assume that the velocity field of each $\hat{\gamma}_{s}$ satisfies the genericity condition (2). Each $\hat{\gamma}_{s}$ has the same length $\tau$ as $\hat{\gamma}$ by the first variation formula. We lift $\hat{\gamma}_{s}$ to a continuous family $\gamma_{s}:[0, \tau] \rightarrow\left(N^{(\prime)}, g^{(\prime)}\right)$ of unit speed geodesics in the universal cover such that $\gamma_{0}(0)=\gamma(0)$, and extend them to geodesics $\gamma_{s}: \mathbb{R} \rightarrow\left(N^{(\prime)}, g^{(\prime)}\right)$; thus $\gamma_{0}=\gamma$. Since the family is continuous, the translational element $\gamma_{s}(\tau) \gamma_{s}(0)^{-1} \in \Gamma^{(\prime)}$ must be constant in $s$, hence equal to $a$ for each $s$. Writing $\dot{\gamma}_{s}(0)=\left(\left(v^{s}, z^{s}\right), V^{s}+Z^{s}\right)$ and $Z^{s}=Z_{c(s)}$, we immediately read off from the first component of $a$ in Lemma 5.4 that $V_{0}^{s} \equiv V_{0}$, whence all $Y_{c(s)}$ are scalar multiples of each other. Moreover, by the lemma, $\tau|c(s)| \in 2 \pi \mathbb{Z}$ is constant. Therefore, $c(s) \equiv c$. By $V_{0}=\beta^{s} Y_{c(s)}=\beta^{s} Y_{c}$ we obtain $\beta^{s} \equiv \beta$. From the coefficient of $Z_{c}$ occurring in the second component of $a$ in Lemma 5.4, we read off that $\left|V_{\perp}^{s}\right| \equiv\left|V_{\perp}\right|$. We continue the discussion separately for the two parts of the statement:

(i) We obtain no restriction at all for the coefficients $y_{i}^{s}, y_{j}^{s}, y_{k}^{s}, z_{i}^{s}, z_{j}^{s}, z_{k}^{s}$ of $v^{s}$ and $z^{s}$. There is also no further restriction on $V_{c_{k}}^{s}$ and $V_{|c|}^{s}$ apart from $\left|V_{c_{k}}^{s}\right|^{2}+\left|V_{|c|}^{s}\right|^{2}=\left|V_{\perp}\right|^{2}$ and (by the genericity condition) the requirement that both vectors be nonzero. These two vectors then determine the coefficients $\alpha_{2}^{s}$ and $\alpha_{4}^{s}$. These, in turn, are by Lemma 5.4 and 
the constancy of $a$ seen to determine $x_{i}^{s} c_{i}+x_{j}^{s} c_{j}$ and $x_{i}^{s} c_{j}-x_{j}^{s} c_{i}$, and thus to determine $x_{i}^{s}$ and $x_{j}^{s}$ (recall that $\left(c_{i}, c_{j}\right) \neq 0$ ).

Note that the sets of points in $\Gamma \cdot(\bar{v}, \bar{z}) \in \Gamma \backslash N$ with fixed $x_{i}, x_{j}$ coordinates for $\bar{v}$ and arbitrary $y_{i}, \ldots, z_{k}$ coordinates are diffeomorphic to $T^{6}$ because the normal subgroup of $N$ whose Lie algebra is spanned by $\left\{Y_{i}, \ldots, Z_{k}\right\}$ is abelian. Moreover, the pairs of possible vectors $V_{c_{k}}^{s}$ and $V_{|c|}^{s}$ fill out the sphere $S^{3}$ with radius $\left|V_{\perp}\right|$ in $\operatorname{span}\left\{V_{0}\right\}^{\perp} \subset \mathfrak{v}$, except for two $S^{1}$ fibers where the first or the second component is zero. If we now drop the genericity condition (i.e., allow $V_{c_{k}}^{s}$ or $V_{|c|}^{s}$ to vanish), the possible pairs of vectors fill out the entire $S^{3}$.

Conversely, it is clear that any initial velocity $\left(\left(v^{s}, z^{s}\right), V^{s}+Z^{s}\right) \in T N$ obtained by choosing the various coordinates according to the restrictions and degrees of freedom described above will indeed correspond to a closed unit speed geodesic in the maximal continuous family containing $\hat{\gamma}$. By the above discussion, this shows that the corresponding submanifold $C$ of the unit tangent bundle is indeed diffeomorphic to $T^{6} \times S^{3}$. (Note that quotienting by $\Gamma$ will not identify pairs of tangent vectors with different $\left(x_{i}, x_{j}\right)$ coordinates of the basepoint because such have been seen to arise only from different velocity vectors.)

The decomposition into the two singular fibers $T^{6} \times S^{1}$ and the one-parameter family of fibers $T^{6} \times S^{1} \times S^{1}$ is respected by the geodesic flow because choosing one of these fibers corresponds to fixing the norm of $\left|V_{c_{k}}^{s}\right|$ (and hence of $\left.\left|V_{|c|}^{s}\right|\right)$ ); note that these norms are invariant under the geodesic flow by 4.2 .

Finally, the $T^{6} \times S^{1} \times S^{1}=T^{8}$ fibers turn out to be level sets of the first eight integrals given in Lemma 4.4: The coefficients $c_{i}, c_{j}, c_{k}$ of $Z$ which are constant here are the values of $q^{Z_{i}}, q^{Z_{j}}, q^{Z_{k}}$. The values $\left|V_{c_{k}}^{s}\right|^{2}$ and $\left|V_{|c|}^{s}\right|^{2}$, which are constant in such a $T^{8}$ fiber, are, up to some multiplicative constants depending on $c$, just the values of $h_{1}$ and $h_{2}$. The value of the first integral $k$ is just $\left\langle V^{s}, Y_{c}\right\rangle=\left\langle\beta Y_{c}, Y_{c}\right\rangle=\beta|c|^{2}$ which is constant as well. Moreover, one straightforwardly computes

$$
\begin{gathered}
v_{\mathfrak{x}}-C(Z) V_{\mathfrak{y}}=x_{i} X_{i}+x_{j} X_{j}-\frac{\alpha_{2}}{c_{k}} E_{1}(Z)-\frac{\alpha_{4}}{|c|} E_{3}(Z) \\
=\left(-\frac{\alpha_{2}}{c_{k}}+\frac{1}{c_{i}^{2}+c_{j}^{2}}\left(x_{i} c_{i}+x_{j} c_{j}\right)\right) E_{1}(Z)+\left(-\frac{\alpha_{4}}{|c|}+\frac{1}{|c|\left(c_{i}^{2}+c_{j}^{2}\right)}\left(x_{i} c_{j}-x_{j} c_{i}\right)\right) E_{3}(Z) .
\end{gathered}
$$

Comparing with the coefficients in the $z$-component of the translational element $a$ in Lemma 5.4, we see that constancy of those coefficients is equivalent to constancy of the first integrals $f^{X_{i}}$ and $f^{X_{j}}$ (note that $\Phi(Z)=\Phi\left(Z_{c}\right)$ is nonzero here because of $c_{k} \neq 0$ ). Conversely, one cannot continuously move out of such a $T^{8}$ fiber without changing the values of any of the first integrals. This finishes the proof of statement (i).

(ii) The discussion is analogous to the corresponding discussion in (i). This time, we obtain no restriction for the coefficients $x_{i}^{s}, x_{j}^{s}, z_{i}^{s}, z_{j}^{s}, z_{k}^{s}$ of $v^{s}$ and $z^{s}$, and the same restrictions vs. freedoms as in (i) for the two vectors $V_{c_{k}}^{s}$ and $V_{|c|}^{s}$. The vector $V_{|c|}^{s}$ then determine the coefficients $\alpha_{3}^{\prime s}$ and $\alpha_{4}^{\prime s}$, which, in turn, determine $y_{k}^{s}-\frac{c_{k}}{c_{i}^{2}+c_{j}^{2}}\left(y_{i}^{s} c_{i}+y_{j}^{s} c_{j}\right)$ and $y_{i}^{s} c_{j}-y_{j}^{s} c_{i}$. The corresponding triples $\left(y_{i}^{s}, y_{j}^{s}, y_{k}^{s}\right)$ constitute an affine line in the $\mathfrak{y}$ component of $\mathfrak{v}=\mathfrak{x} \oplus \mathfrak{y}$ whose direction is rational with respect to the sublattice of $\Gamma^{\prime}$ 
spanned by $Y_{i}, Y_{j}, Y_{k}$ : Note that $c$ must be a scalar multiple of a rational vector because $\tau V_{0}=\tau \beta Y_{c}$ was by Lemma 5.4 the first component of $a \in \Gamma^{\prime}$.

The sets of points in $\Gamma^{\prime} \cdot(\bar{v}, \bar{z}) \in \Gamma^{\prime} \backslash N^{\prime}$ with fixed $y_{i}, y_{j}, y_{k}$ coordinates for $\bar{v}$ and arbitrary $x_{i}, x_{j}, z_{i}, z_{j}, z_{k}$ coordinates are diffeomorphic to $H^{3} \times T^{2}$, where $H^{3}$ is a compact three-dimensional Heisenberg manifold, corresponding to the three-dimensional Heisenberg algebra spanned by $\left\{X_{i}, X_{j}, Z_{k}\right\}$ in $\mathfrak{n}^{\prime}$. Moreover, the pairs of possible vectors $V_{c_{k}}^{s}$ and $V_{|c|}^{s}$ have exactly the same freedom as in (i) and fill out, after dropping the genericity condition on these, the sphere $S^{3}$ of radius $\left|V_{\perp}\right|$ in $\operatorname{span}\left\{V_{0}\right\}^{\perp}$. Finally, the affine line of possible $\left(y_{i}^{s}, y_{j}^{s}, y_{k}^{s}\right)$ determined via $\alpha_{3}^{\prime s}$ and $\alpha_{4}^{\prime s}$ by these vectors yields another $S^{1}$ factor after quotienting by $\Gamma^{\prime}$ (recall that the line had rational direction); this indeed splits off as a factor because $\mathfrak{y}$ commutes with $\mathfrak{x} \oplus \mathfrak{z} \subset \mathfrak{n}^{\prime}$.

By analogous arguments as in (i), we conclude that $C^{\prime}$ is indeed diffeomorphic to $H^{3} \times S^{1} \times T^{2} \times S^{3}=H^{3} \times T^{3} \times S^{3}$, decomposed as claimed in the statement.

Remark 5.7. We do not present here the corresponding analysis for initial velocities which do not satisfy the genericity condition (2). We just mention that the submanifolds of the unit tangent bundle fibered by closed geodesics belonging to central free homotopy classes but having noncentral velocity fields (this is the case for most of such geodesics) are of dimension ten for both of the manifolds from Example 2.6. There are other special free homotopy classes for which dimensions lower than eight occur.

Corollary 5.8. For both of the 8-dimensional manifolds from Example 2.6, the set of initial velocity vectors of closed unit speed geodesics satisfying the genericity condition (2) is dense in the unit tangent bundle. In particular, by Corollary 5.6: The $T^{8}$ fibers of the unit tangent bundle of the first manifold $(\Gamma \backslash N, g)$ which are invariant under the geodesic flow and are level sets of the eight first integrals from Lemma 4.4 are dense in the unit tangent bundle; the same holds for the corresponding invariant $H^{3} \times T^{5}$ fibers in the unit tangent bundle of the second manifold $\left(\Gamma^{\prime} \backslash N^{\prime}, g^{\prime}\right)$.

Proof. We carry out the proof for $(\Gamma \backslash N, g)$ using formula (44) from Lemma 5.4 the proof for $\left(\Gamma^{\prime} \backslash N^{\prime}, g^{\prime}\right)$ is completely analogous, using formula (5) instead.

Let $((\bar{v}, \bar{z}), \bar{V}+\bar{Z})$ be an arbitrary element in the unit tangent bundle of $(N, g)$. Write $\bar{Z}=Z_{\bar{c}}$ for some $\bar{c} \in \mathbb{R}^{3}$. Let $\varepsilon>0$ be arbitrary. Choose $c \in \mathbb{Q}^{3}$ such that $|c-\bar{c}|<\varepsilon$, $|c|>\left|c_{k}\right|>0$, and $c_{k} /|c| \in \mathbb{Q}$. For the latter condition, note that the rational points $c$ with rational value of $c_{k} /|c|$ are dense in $\mathbb{R}^{3}$ because the rational points are dense in the unit sphere (since the standard stereographic projection preserves rationality). Choose $\sigma>0$ such that $\sigma c_{k}$ and $\sigma|c|$ are in $2 \pi \mathbb{Z}$ (for instance, $\sigma:=2 \pi \frac{q}{|c|}$ if $c_{k} /|c|=p / q$ with $p \in \mathbb{Z}, q \in \mathbb{N})$. Let $Z:=Z_{c}$ and write $\bar{V}=\bar{V}_{0}+\bar{V}_{\perp}$ with $\bar{V}_{0} \in \operatorname{span}\left\{Y_{c}\right\}$ and $\bar{V}_{\perp} \perp Y_{c}$. Choose $0 \neq V_{0} \in \operatorname{span}\left\{Y_{c}\right\}$ such that $\left|V_{0}-\bar{V}_{0}\right|<\varepsilon$ and $V_{0} \in \frac{1}{\sigma} \mathbb{Q} Y_{c}$. Choose $V_{\perp} \perp Y_{c}$ such that $\left|V_{\perp}-\bar{V}_{\perp}\right|<\varepsilon, V_{\perp}$ is not orthogonal to neither $\operatorname{span}\left\{E_{1}(Z), E_{2}(Z)\right\}$ nor $\operatorname{span}\left\{E_{3}(Z), E_{4}(Z)\right\}$, and such that $\left|V_{\perp}\right|^{2} \in \frac{1}{\sigma} \mathbb{Q}$. Let $V:=V_{0}+V_{\perp}$. Finally, choose $v \in \mathfrak{v}$ such that $|v-\bar{v}|<\varepsilon$ and

$\beta\left(\alpha_{2}-\frac{c_{k}}{c_{i}^{2}+c_{j}^{2}}\left(x_{i} c_{i}+x_{j} c_{j}\right)\right) \in \frac{1}{\sigma} \mathbb{Q}$ and $-\frac{\left|V_{c_{k}}\right|^{2}}{2 c_{k}|c|^{2}}+\beta\left(\alpha_{4}-\frac{1}{c_{i}^{2}+c_{j}^{2}}\left(x_{i} c_{j}-x_{j} c_{i}\right)\right) \in \frac{1}{\sigma} \mathbb{Q}$, 
where $V_{0}=\beta Y_{c}, V_{\perp}=\sum_{m=1}^{4} \alpha_{m} E_{m}(Z)$, and $v=x_{i} X_{i}+x_{j} X_{j}+y_{i} Y_{i}+y_{j} Y_{j}+y_{k} Y_{k}$. Let $\bar{z}:=z$. Then by Lemma 5.4, for the geodesic $\gamma$ with initial velocity $((v, z), V+Z)$ we have $\gamma(\sigma) \gamma(0)^{-1} \in \exp \left(\operatorname{span}_{\mathbb{Q}}\left\{Y_{i}, \ldots, Z_{k}\right\}\right)$. By replacing $\sigma$ with a suitable multiple $\tau=m \sigma$, where $m \in \mathbb{N}$, we obtain $a:=\gamma(\tau) \gamma(0)^{-1} \in \Gamma$ and (still) $\tau c_{k} \in 2 \pi \mathbb{Z}, \tau|c| \in 2 \pi \mathbb{Z}$. In particular, $e^{\tau j(Z)}=\operatorname{Id}_{\mathfrak{v}}$ and hence $L_{a *} \dot{\gamma}(0)=\dot{\gamma}(\tau)$, thus $\left.\gamma\right|_{[0, \tau]}$ descends to a $\tau$-periodic geodesic in $(\Gamma \backslash N, g)$. Summarizing, we have shown: Arbitrarily close to any unit tangent vector $((\bar{v}, \bar{z}), \bar{V}+\bar{Z})$, we find another tangent vector $((v, z), V+Z)$ satisfying the genericity condition (2) corresponding to the initial vector of a closed geodesic in $(\Gamma \backslash N, g)$. Choosing a sequence of such vectors converging to $((\bar{v}, \bar{z}), \bar{V}+\bar{Z})$ and normalizing each vector of this sequence, we obtain a sequence of unit vectors converging to our given unit vector, consisting of initial velocities of closed geodesics and satisfying the genericity condition (2). This proves the statement.

Remark 5.9. On the one hand, it seems surprising at first sight that two manifolds with the same Laplace spectrum, thus with the same wave trace, can differ so radically with respect to the behaviour of their geodesic flows. On the other hand, as explained in the Introduction, the fact that continuous families of closed geodesics in our pair of manifolds have been seen to fill out larger dimensional families than Lagrangian tori shows that the suitable version of the condition of "clean simple length spectrum", under which the problem of audibility of complete integrability and any positive conjecture in this respect would make most sense, is violated here anyway. This yields an explanation of why isospectrality in these examples fails to entail similar integrability properties.

An even more obvious explanation would be provided if the two manifolds failed to satisfy the so-called Clean Intersection Hypothesis, a condition always needed for even establishing a wave trace formula; see [3]. However, it turns out that both of our manifolds actually do satisfy the Clean Intersection Hypothesis, which is defined as the following condition: For any number $\ell>0$ and any free homotopy class $\alpha$ containing a closed geodesic of length $\ell$, the subset $W_{\ell}(\alpha)$ of the unit tangent bundle consisting of the velocity vectors of all unit speed closed geodesics of length $\ell$ and contained in $\alpha$ is a finite union of submanifolds of the unit tangent bundle, and for the differential of the time- $\ell$-map of the geodesic flow at any point of $W_{\ell}(\alpha)$, the eigenspace associated with the eigenvalue 1 is not larger than the tangent space of $W_{\ell}(\alpha)$ at that point.

In fact, Ruth Gornet [4] has recently shown that a compact, two-step Riemannian nilmanifold $(\Gamma \backslash N, g)$ satisfies this hypothesis if and only if, using the notation from 2.1 and 2.2, for all $V+Z \in \exp ^{-1}(\Gamma) \subset \mathfrak{n}$ and all nonzero eigenvalues $\pm i \vartheta$ of $j\left(\operatorname{proj}_{[V, \mathfrak{n}] \perp} Z\right)$ (where proj denotes orthogonal projection) we have $\vartheta \notin \pi \mathbb{Q}$. In our manifolds from Example 2.6, let us endow $\mathfrak{v}$ and $\mathfrak{z}$ with the rational structure given by $\operatorname{span}_{\mathbb{Q}}\left\{X_{i}, \ldots, Y_{k}\right\}$ and $\operatorname{span}_{\mathbb{Q}}\left\{Z_{i}, Z_{j}, Z_{k}\right\}$. Then for all $V+Z \in \exp ^{-1}\left(\Gamma^{(\prime)}\right), V$ and $Z$ are rational vectors by our definition of $\Gamma^{(\prime)}$. The subspace $[V, \mathfrak{n}]=[V, \mathfrak{v}] \subseteq \mathfrak{z}$ is then rational as well. Elementary arguments (using the orthonormality of the rational basis $\left\{Z_{i}, Z_{j}, Z_{k}\right\}$ ) show that the orthogonal projection of the rational vector $Z$ to this rational subspace is again a rational vector. Denote the resulting vector by $Z_{V}^{\perp}=c_{i} Z_{i}+c_{j} Z_{j}+c_{k} Z_{k}$. Then $c_{i}, c_{j}, c_{k}$ are rational; in particular, the possibly nonzero eigenvalues $-c_{k}^{2}$ and $-|c|^{2}$ of $j\left(Z_{V}^{\perp}\right)^{2}$ are rational. So 
these can, if nonzero, never be in $\pi^{2} \mathbb{Q}$. This implies that Gornet's necessary and sufficient criterion for the Clean Intersection Hypothesis is indeed satisfied.

\section{REFERENCES}

[1] Butler, Leo T. Integrable geodesic flows with wild first integrals: the case of two-step nilmanifolds. Ergodic Theory Dynam. Systems 23 (2003), no. 3, 771-797; MR 1992663.

[2] Butler, Leo T. Zero entropy, non-integrable geodesic flows and a non-commutative rotation vector. Trans. Amer. Math. Soc. 355 (2003), no. 9, 3641-3650; MR 1990166.

[3] Duistermaat, H., Guillemin, V. The spectrum of positive elliptic operators and periodic bicharacteristics. Invent. Math. 29 (1975), no. 1, 39-79; MR 0405514.

[4] Gornet, R. Riemannian nilmanifolds and the trace formula. Trans. Amer. Math. Soc. 357 (2005), no. 11, 4445-4479; MR 2156717.

[5] Eberlein, P. Geometry of two-step nilpotent Lie groups with a left invariant metric. Ann. Sci. de l'Ecole Norm. Sup. 27 (1994), 611-660; MR 1296558.

[6] Gordon, C., Schueth, D., Mao, Y. Symplectic rigidity of geodesic flows on two-step nilmanifolds. Ann. Sci. Ecole Norm. Sup. (4) 30 (1997), no. 4, 417-427; MR 1456241.

[7] Gordon, C., Wilson, E. Isospectral deformations of compact solvmanifolds. J. Differential Geom. 19 (1984), no. 1, 241-256; MR 0739730.

[8] Gordon, C., Wilson, E. Continuous families of isospectral Riemannian manifolds which are not locally isometric. J. Diff. Geom. 47 (1997), 504-529; MR 1617640.

[9] Taimanov, I. A. Topological obstructions to the integrability of geodesic flows on nonsimply connected manifolds. (Russian) Izv. Akad. Nauk SSSR Ser. Mat. 51 (1987), no. 2, 429-435, 448; translation in Math. USSR-Izv. 30 (1988), no. 2, 403-409; MR 0897007.

[10] Zelditch, S. Wave invariants for nondegenerate closed geodesics. Geom. Funct. Anal. 8 (1998), 179202; MR 1601862.

[11] Zelditch, S. The inverse spectral problem. In: Surveys in Differential Geometry IX (2004), Int. Press, Somerville, MA.

Institut für Mathematik, Humboldt-Universität Zu Berlin, D-10099 Berlin, Germany

E-mail address: schueth@math.hu-berlin.de 